\title{
BMJ Open The relationship between mental health and risk of active tuberculosis: a systematic review
}

\author{
Sally E Hayward (D) , ${ }^{1,2}$ Anna Deal (D) , ${ }^{1,2}$ Kieran Rustage (D) , 1 \\ Laura B Nellums (D) , ${ }^{1,3}$ Annika C Sweetland, ${ }^{4}$ Delia Boccia, ${ }^{2}$ Sally Hargreaves (D) , ${ }^{1}$ \\ Jon S Friedland (i) ${ }^{1}$
}

To cite: Hayward SE, Deal A, Rustage K, et al. The relationship between mental health and risk of active tuberculosis: a systematic review. BMJ Open 2022;12:e048945. doi:10.1136/ bmjopen-2021-048945

- Prepublication history and additional supplemental material for this paper are available online. To view these files, please visit the journal online (http://dx.doi.org/10.1136/ bmjopen-2021-048945).

DB, SH and JSF are joint senior authors.

Received 11 January 2021 Accepted 06 October 2021

Check for updates

(C) Author(s) (or their employer(s)) 2022. Re-use permitted under CC BY. Published by BMJ.

${ }^{1}$ Institute for Infection and Immunity, St George's, University of London, London, UK

${ }^{2}$ Faculty of Public Health and Policy, London School of Hygiene and Tropical Medicine, London, UK

${ }^{3}$ Division of Epidemiology and Public Health, School of Medicine, University of Nottingham, Nottingham, UK ${ }^{4}$ Department of Psychiatry, Columbia Vagelos College of Physicians and Surgeons, New York State Psychiatric Institute, New York, New York, USA

Correspondence to

Sally E Hayward;

shayward@sgul.ac.uk

\section{ABSTRACT}

Objectives Tuberculosis (TB) and mental illnesses are highly prevalent globally and often coexist. While poor mental health is known to modulate immune function, whether mental disorders play a causal role in TB incidence is unknown. This systematic review examines the association between mental health and TB disease risk to inform clinical and public health measures.

Design Systematic review, following Preferred Reporting Items for Systematic Reviews and Meta-Analyses (PRISMA) guidelines.

Search strategy and selection criteria MEDLINE, PsycINFO and PsycEXTRA databases were searched alongside reference list and citation searching. Inclusion criteria were original research studies published 1 January 1970-11 May 2020 reporting data on the association between mental health and TB risk.

Data extraction, appraisal and synthesis Data were extracted on study design and setting, sample characteristics, measurement of mental illness and TB, and outcomes including effect size or prevalence. Studies were critically appraised using Critical Appraisal Skills Programme (CASP) and Appraisal Tool for Cross-Sectional Studies (AXIS) checklists.

Results 1546 records published over 50 years were screened, resulting in 10 studies included reporting data from 607184 individuals. Studies span across Asia, South America and Africa, and include mood and psychotic disorders. There is robust evidence from cohort studies in Asia demonstrating that depression and schizophrenia can increase risk of active TB, with effect estimates ranging from $\mathrm{HR}=1.15(95 \% \mathrm{Cl} 1.03$ to 1.28$)$ to $2.63(95 \% \mathrm{Cl} 1.74$ to 3.96$)$ for depression and $\mathrm{HR}=1.52(95 \% \mathrm{Cl} 1.29$ to 1.79) to $R R=3.04$ for schizophrenia. These data align with evidence from cross-sectional studies, for example, a large survey across low-income and middle-income countries $(n=242952)$ reports $0 R=3.68(95 \% \mathrm{Cl} 3.01$ to 4.50$)$ for a depressive episode in those with TB symptoms versus those without.

Conclusions Individuals with mental illnesses including depression and schizophrenia experience increased TB incidence and represent a high-risk population to target for screening and treatment. Integrated care for mental health and TB is needed, and interventions tackling mental illnesses and underlying drivers may help reduce TB incidence globally.

PROSPERO registration number CRD42019158071.
Strengths and limitations of this study

- This review examines all available evidence on the relationship between mental health and tuberculosis (TB) incidence holistically.

- Comprehensive systematic review methods were used, following Preferred Reporting Items for Systematic Reviews and Meta-Analyses (PRISMA) guidelines.

- There was considerable variation in study design, which limits comparability of results across the included studies.

\section{INTRODUCTION}

Both tuberculosis (TB) and mental health are urgent global health priorities, with 1.4 million TB deaths worldwide in $2019,{ }^{1}$ and approximately $14 \%$ of the global burden of disease attributable to neuropsychiatric disorders. ${ }^{2}$ There is increasing recognition that physical and mental health are interconnected. ${ }^{34}$ Mental illnesses are highly prevalent among TB patients and vice versa, ${ }^{56}$ and poor mental health is associated with reduced treatment-seeking and adherence, and therefore with greater morbidity, mortality, transmission and drug resistance. ${ }^{7}$ Moreover, TB can infect the central nervous system (CNS) causing neurological symptoms, ${ }^{9}$ and certain anti-TB medications have psychiatric side effects. ${ }^{5}$ The greatest burden of TB is experienced in individuals with risk factors including homelessness, drug and alcohol misuse, and migration. ${ }^{10}$ Mental illness is more prevalent in all of these groups. ${ }^{11-13}$ The relationships between TB and mental health are highly complex, with TB-depression comorbidity termed a 'syndemic' due to the bidirectional synergies involved. ${ }^{14}$

Previous research has examined the impact of TB and its treatment on mental health, ${ }^{5}$ the relationship between mental disorders and TB treatment outcomes ${ }^{715}$ and adherence, ${ }^{816}$ 
and the prevalence of mental disorders among $\mathrm{TB}$ and multidrug-resistant TB (MDR-TB) patients. ${ }^{17-19}$ There is increasing interest in whether mental health may increase TB risk, supported by a growing body of evidence that chronic stressors and poor mental health directly influence the immune system, including susceptibility to infection. ${ }^{14}{ }^{20}$ How these pathways operate in TB is unknown, although various mechanisms are possible; for instance, the suppression of cellular immunity due to poor mental health could contribute to reactivation of latent TB infection (LTBI) or progression from subclinical to clinical disease. ${ }^{21}$ However, while the bidirectional relationship between mental health and TB has been the subject of discussion, ${ }^{61421-25}$ there has been no systematic and comprehensive examination of the evidence base on associations between mental health and TB incidence. This systematic review aims to examine the evidence on the relationship between mental health and $\mathrm{TB}$, to provide insight into whether poor mental health may be a risk factor for TB disease and to inform clinical and global public health measures.

\section{METHODS}

\section{Search strategy}

We carried out a systematic review following Preferred Reporting Items for Systematic Reviews and MetaAnalyses (PRISMA) guidelines, registered with PROSPERO (CRD42019158071) ${ }^{26}$. MEDLINE, PsycINFO and PsycEXTRA were searched from inception to 11 May 2020, combining terms and subject headings for mental health and TB (online supplemental box 1), restricting the search to English language papers. Records were imported into EndNote, and duplicates were deleted. Two independent reviewers (SEH and KR/AD) carried out title/abstract and full-text screening using Rayyan QCRI. ${ }^{27}$ We searched reference lists and carried out citation searching via Web of Science for included papers and previous reviews in this area. ${ }^{57815-19}$

\section{Selection criteria}

This study includes empirical research reporting on the relationship between poor mental health and risk of active TB (table 1). This includes two study designs: (1) longitudinal studies investigating the causal relationship between mental health and TB incidence and (2) cross-sectional studies investigating the association between mental health and TB disease. Cross-sectional studies were included regardless of whether they treated mental health as the exposure and TB as the outcome or vice versa, since both provide evidence for an association (provided that those with a history of mental illness are not excluded). Prevalence studies were excluded, as studies without controls cannot establish the presence of an association. No exclusions were made based on population, age or geographic location.

The exposure measure was mental ill health, assessed either as an overall measure of mental illness or as a specific mental disorder. Mental disorders are defined here to include psychotic disorders (eg, schizophrenia), mood or affective disorders (eg, depression), and neurotic, stress-related and somatoform disorders (eg, anxiety). ${ }^{28}$ Mental illness may be diagnosed clinically (ie, by a medical professional and/or prescription of

\begin{tabular}{|c|c|c|}
\hline & Inclusion criteria & Exclusion criteria \\
\hline Population & $\begin{array}{l}\text { Any population in any geographic } \\
\text { location, including vulnerable groups, } \\
\text { for example, the homeless }\end{array}$ & \\
\hline Exposure & $\begin{array}{l}\text { Mental illness, including psychotic } \\
\text { and affective disorders, diagnosed } \\
\text { clinically or by any psychological tool }\end{array}$ & $\begin{array}{l}\text { Alcohol and drug disorders. } \\
\text { Studies where mental illness is not a primary variable. }\end{array}$ \\
\hline Control & No mental illness & \\
\hline Outcome & $\begin{array}{l}\text { Incidence of active TB in humans, } \\
\text { including reactivation of LTBI and } \\
\text { MDR-TB }\end{array}$ & $\begin{array}{l}\text { LTBI without reactivation. } \\
\text { CNS TB (including tuberculosis meningitis). } \\
\text { TB-HIV coinfection. } \\
\text { Studies investigating outcomes, for example, mortality (and not incidence). }\end{array}$ \\
\hline $\begin{array}{l}\text { Study } \\
\text { design }\end{array}$ & $\begin{array}{l}\text { Observational epidemiological studies } \\
\text { (a) longitudinal studies (cohort): } \\
\text { mental health must be treated as the } \\
\text { exposure and TB as the outcome; } \\
\text { (b) cross-sectional studies (cross- } \\
\text { sectional and case-control): mental } \\
\text { health may be treated as the exposure } \\
\text { and TB as the outcome or vice versa }\end{array}$ & $\begin{array}{l}\text { Prevalence studies without controls. } \\
\text { Qualitative studies. } \\
\text { Case reports. } \\
\text { Non-original research, for example, protocols, commentaries, reviews. } \\
\text { Longitudinal studies in which TB is the exposure and mental health is the } \\
\text { outcome. }\end{array}$ \\
\hline Dates & Papers published 1970-2020 & \\
\hline
\end{tabular}

CNS, central nervous system; LTBI, latent tuberculosis infection; MDR-TB, multi-drug resistant tuberculosis; PECOS, population, exposure, control, outcome, study design; TB, tuberculosis. 


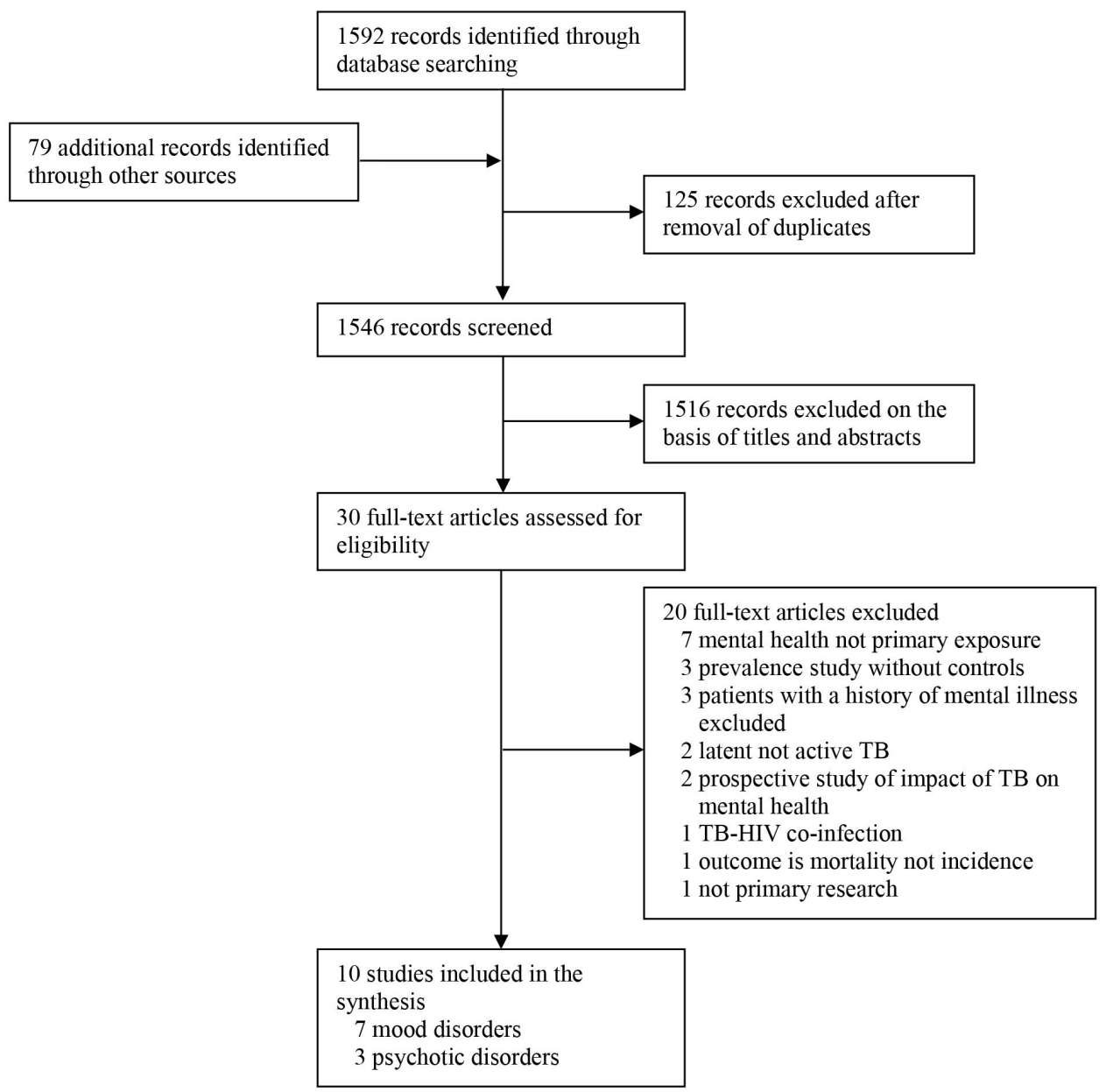

Figure 1 Study selection, PRISMA flow chart. PRISMA, Preferred Reporting Items for Systematic Reviews and Meta-Analyses; TB, tuberculosis.

medication) or by any psychological tool (ie, a structured questionnaire or interview). Only studies for which mental illness was a primary variable were included to ensure that the study was powered to detect an association. Studies focused solely on substance use disorders were excluded as alcohol and drug use are already known to be strongly associated with $\mathrm{TB}$ risk, possibly due to social mixing patterns, or the effect of these substances on the immune system. ${ }^{29-31}$

The outcome measure was active TB disease, diagnosed according to bacteriological, clinical and/or radiological criteria. ${ }^{32}$ This excludes LTBI but includes reactivated disease. TB of any site, pulmonary or extrapulmonary, was included, with the exception of CNS TB. TB-HIV coinfection was also excluded. In these cases, different mechanisms are expected to underlie the associations. Studies relating to outcomes of TB infection or treatment (including psychiatric side effects of anti-TB medication), rather than incidence, were excluded, as this has been previously reviewed. $^{715}$

\section{Data extraction}

Two independent reviewers ( $\mathrm{SEH}$ and $\mathrm{KR} / \mathrm{AD}$ ) extracted data using a standardised form adapted from the Cochrane Effective Practice and Organisation of Care
(EPOC) group, ${ }^{33}$ including study characteristics, population, setting, methods, participants, exposure, outcome, results (summary estimates), and applicability.

\section{Critical appraisal}

Two independent reviewers (SEH and KR/AD) assessed the quality of all included studies, using Critical Appraisal Skills Programme (CASP) checklists for cohort and casecontrol studies and the Appraisal Tool for Cross-Sectional Studies (AXIS) tool for cross-sectional studies. ${ }^{34} 35$ The tools were amended to remove questions on local applicability and implications for practice, instead asking about external validity and implications for the review. A study was defined as high quality at a critical appraisal score of above $90 \%$, moderate above $60 \%$ and low at $60 \%$ or below. This score relates to the appropriateness and relevance of the study for this review question rather than in relation to the study's own aims.

\section{Synthesis}

Extracted data were tabulated, detailing methods and results of included studies, categorised by type of mental illness. Results are presented as reported in the studies. The primary outcome extracted is the main effect measure for the relationship between mental health and TB, 


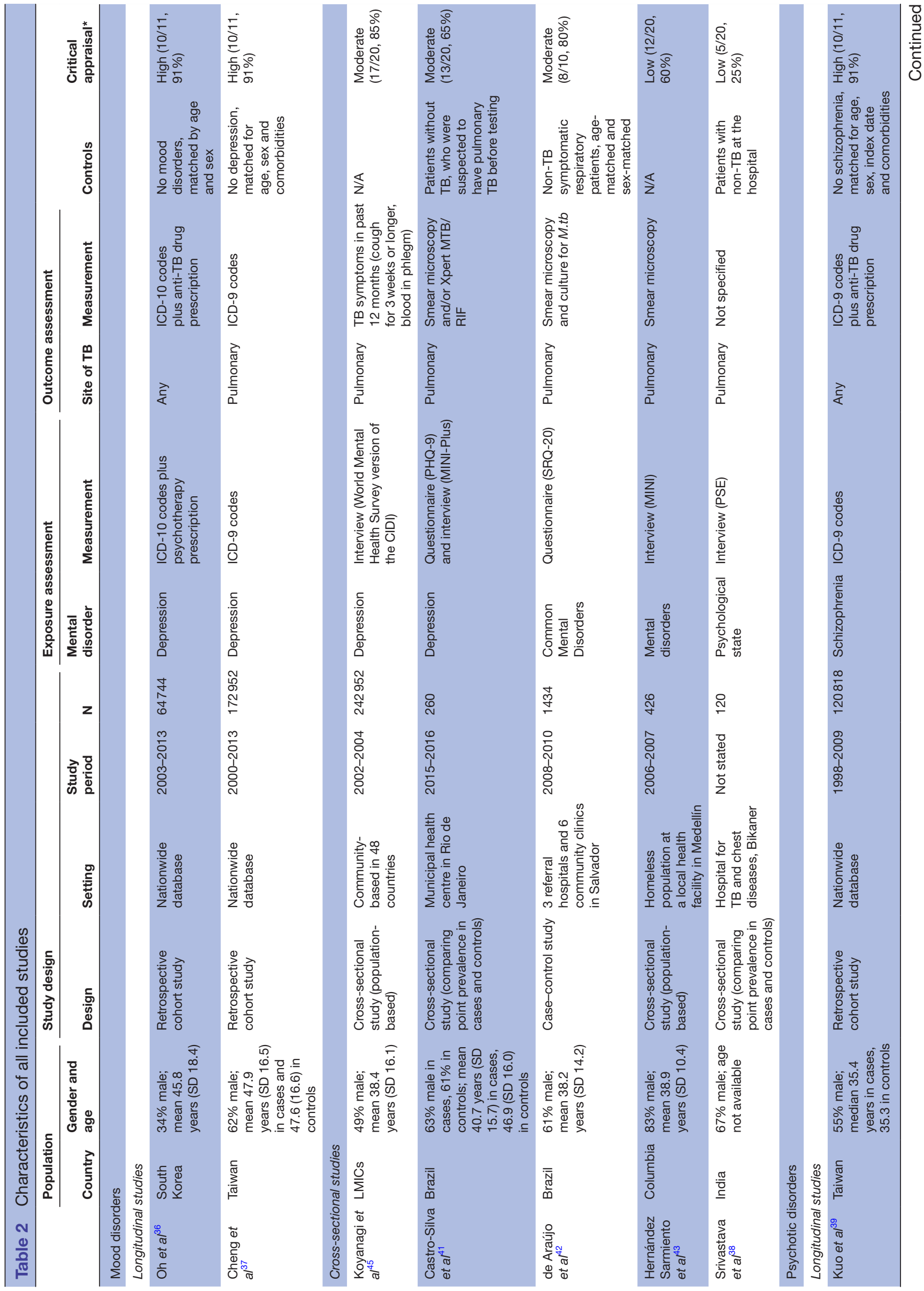




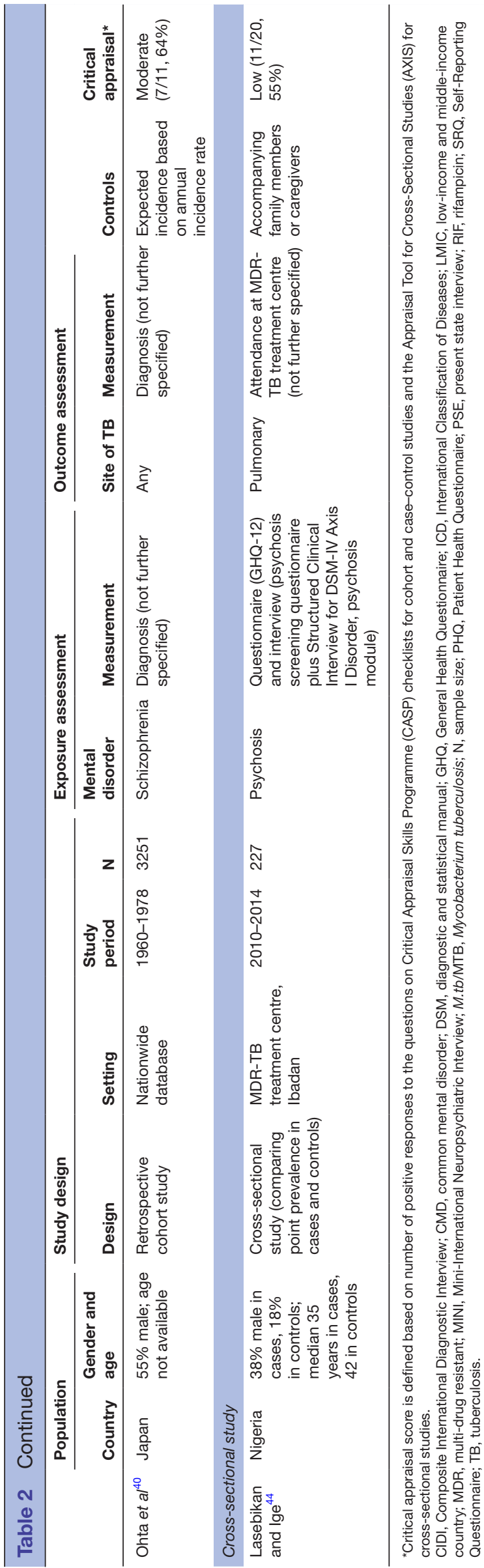

and the secondary outcome is any additional measures reported relating to different definitions or levels of mental illness. For cross-sectional studies, any cases of mental illness arising post-TB diagnosis or treatment were excluded where possible. Given the wide range of populations, study designs and statistical methods used, meta-analysis was not appropriate. A narrative synthesis was therefore completed, by study design and type of mental illness. Effect size and precision were compared where studies were sufficiently similar. The evidence as a whole was assessed as to whether poor mental health may be associated with, and act as a risk factor for, TB disease.

\section{Patient and public involvement}

Patients and/or the public were not involved in the design, conduct, reporting or dissemination plans of this research.

\section{RESULTS}

We screened 1546 records published over 50 years and found that 10 published articles met the inclusion criteria (figure 1), with a combined sample size of 607184 . Reasons for exclusion are presented in figure 1, with the most common being that mental health is not a primary outcome or that the study design does not meet the inclusion criteria outlined above.

Included studies span multiple locations, including five in Asia, ${ }^{36-40}$ three in South America, ${ }^{41-43}$ one in Africa ${ }^{44}$ and one across low-income and middle-income countries (LMICs). ${ }^{45}$ Two studies were published in the $1980 \mathrm{~s}^{38} 40$ and the remainder within the last 10 years. ${ }^{36373941-45}$ Seven studies assess mood disorders ${ }^{36-38} 41-4345$ and three assess psychotic disorders. ${ }^{39} 4044$ Seven studies investigate pulmonary TB exclusively, ${ }^{38} 38{ }^{41-45}$ and three consider both pulmonary and extrapulmonary disease. ${ }^{3694}$ Study characteristics are summarised in table 2. Only one paper included here was included in any prior systematic review of mental health and TB. ${ }^{57815-19}$

Several study designs were used to investigate the association between mental health and TB. Four retrospective cohort studies use insurance or registry data to follow-up TB incidence in those with a mental illness compared with matched controls ${ }^{36} 37$ or general population incidence. ${ }^{40}$ Where specified, the studies use International Classification of Diseases (ICD-9 or ICD-10) codes on patient medical records, in addition to the prescription of medication, to identify patients with a mental disorder and with TB disease. These are large studies, making up $59.6 \%$ of the total sample size $(\mathrm{n}=361765)$ (figure 2$)$.

Other studies use questionnaire screening tools or interviews to assess mental health at a single timepoint. These may be short disorder-specific questionnaires, such as the Patient Health Questionnaire-9 (PHQ-9) for depression, ${ }^{46}$ or longer interviews covering a range of psychiatric disorders, such as the Composite International Diagnostic Interview (CIDI) ${ }^{47}$ One case-control study identifies mental illnesses prior to TB diagnosis in cases and 


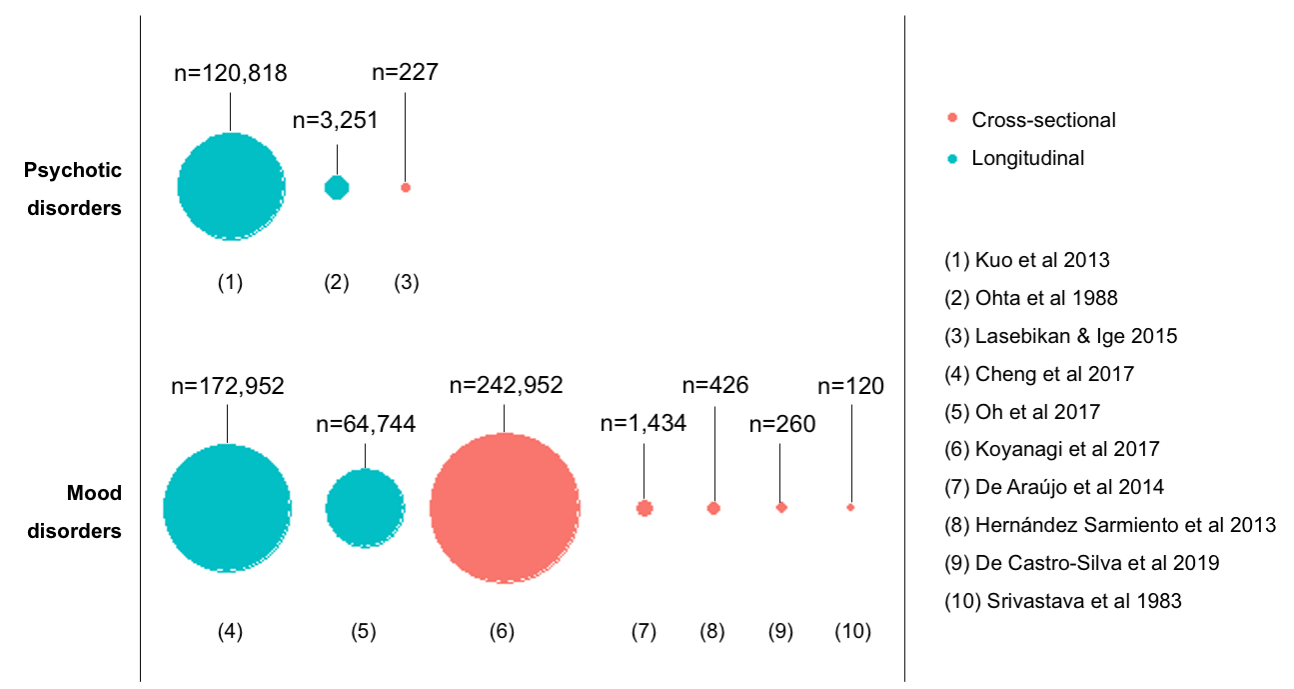

Figure 2 Sample size of included studies.

matched controls. ${ }^{42}$ Two population-based cross-sectional studies establish prevalence of mental illnesses and TB, and assess the association between them. ${ }^{43}$ Three additional cross-sectional studies compare point prevalence of mental illnesses in TB cases and controls. ${ }^{38} 4144$ One of the population-based cross-sectional studies is very large, making up $40.0 \%$ of the total sample size $(\mathrm{n}=242952)$, whereas the other five cross-sectional studies are smaller, making up $0.4 \%$ of the total sample size $(n=2467)$ (figure 2).

While the retrospective cohort studies use populationbased databases or registers, ${ }^{36} 373940$ other studies are only applicable to certain populations such as the homeless patients ${ }^{43}$ or patients with MDR-TB. ${ }^{44}$ Included studies vary in quality, with three high, four moderate and three low-quality studies overall, and an average critical appraisal score of $71 \%$ for mood and $70 \%$ for psychotic disorder papers. The longitudinal studies are all moderate to high quality (average score $84 \%$ ), whereas the cross-sectional studies are low to moderate quality (average score 62\%). Common problems identified are issues with sampling and inadequate consideration of confounding. Results and study quality are tabulated in table 3 and in detail in online supplemental tables 1,2.

\section{Mood disorders}

Four studies specifically investigate depression, 36374145 and three look generally at mood disorders. ${ }^{38} 4243$ This includes two large, high-quality cohort studies on depression, both using nationally representative databases. One, in South Korea, reports an adjusted HR for TB incidence among those with depression compared with controls of 2.63 (95\% CI 1.74 to $3.96, \mathrm{p}<0.001),{ }^{36}$ and the other, in Taiwan, reports an adjusted HR of 1.15 (95\% CI 1.03 to 1.28). ${ }^{37}$ The former also finds a dose-response relationship, with more severe depression associated with greater TB risk. Both studies report a higher TB incidence rate in those with depression than in controls (figure 3).
Other studies use a cross-sectional design to assess the association between mood disorders and TB. A large study using community-based World Health Survey data from across LMICs reports an adjusted OR for a depressive episode in those with TB symptoms vs those without of 3.68 (95\% CI 3.01 to 4.50$).{ }^{45}$ By contrast, a cross-sectional study among presumptive pulmonary TB cases in Rio de Janeiro, Brazil that compares the prevalence of depression among patients with and without a bacteriologically confirmed diagnosis of pulmonary TB finds no evidence of a difference. Of 259 participants screened for depression, the prevalence among TB cases is $60.2 \%$ versus $62.1 \%$ in controls (crude OR $=0.92,95 \%$ CI 0.55 to 1.54 , $\mathrm{p}=0.79$ ), and of 159 who screened positive for depression, this diagnosis was confirmed in $59.5 \%$ of $\mathrm{TB}$ cases versus $50.9 \%$ of controls (crude OR=1.42, 95\% CI 0.63 to 3.19, $\mathrm{p}=0.42){ }^{41}$

Beyond depression, a case-control study in Salvador, Brazil considers common mental disorders (CMDs) more broadly, characterised by diverse depressive, anxiety or somatoform symptoms, and finds elevated odds of having TB among those with a CMD (adjusted OR $=1.34,95 \%$ CI 1.05 to 1.70$).{ }^{42}$ A cross-sectional study among the homeless in Medellín, Columbia investigates the association between TB and various psychiatric illnesses, but finds that only dysthymia (persistent mild depression) and history of major depression are associated with $\mathrm{TB}$, and only for dysthymia is this statistically significant in multivariate analysis (adjusted $\mathrm{OR}=2.54,95 \%$ CI 1.10 to 5.86, $\mathrm{p}=0.028) .{ }^{43}$ Finally, a cross-sectional study into the current psychological state of TB cases and controls in Bikaner, India finds a significant association between TB and symptoms including anxiety, depressed mood and sleep/appetite disturbances, and reports a prevalence of psychiatric illness in TB patients of $41.6 \%$ versus $13.3 \%$ in controls. ${ }^{38}$

\section{Psychotic disorders}

Two studies specifically investigate schizophrenia, ${ }^{3940}$ and one considers psychosis more generally. ${ }^{44}$ 


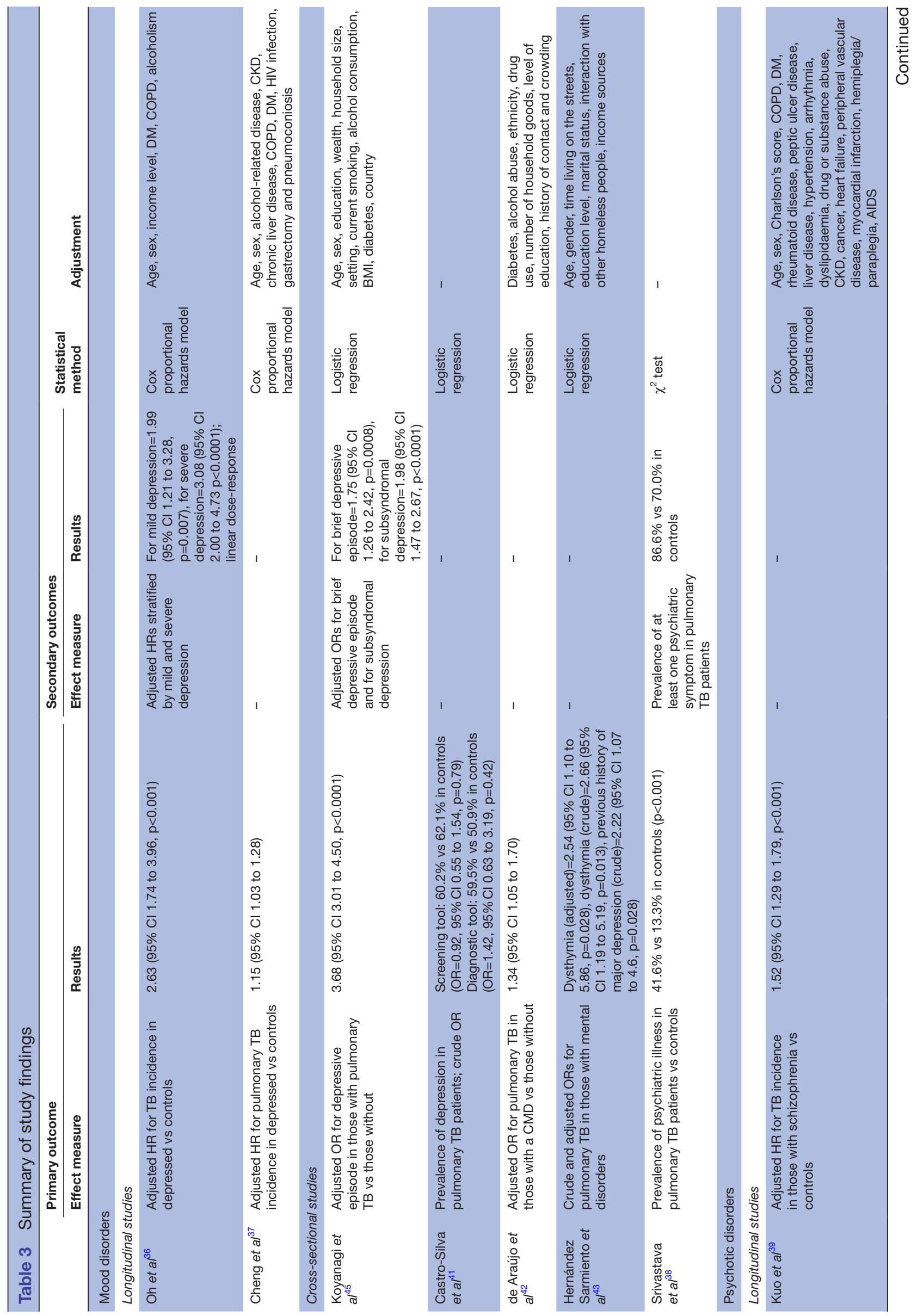




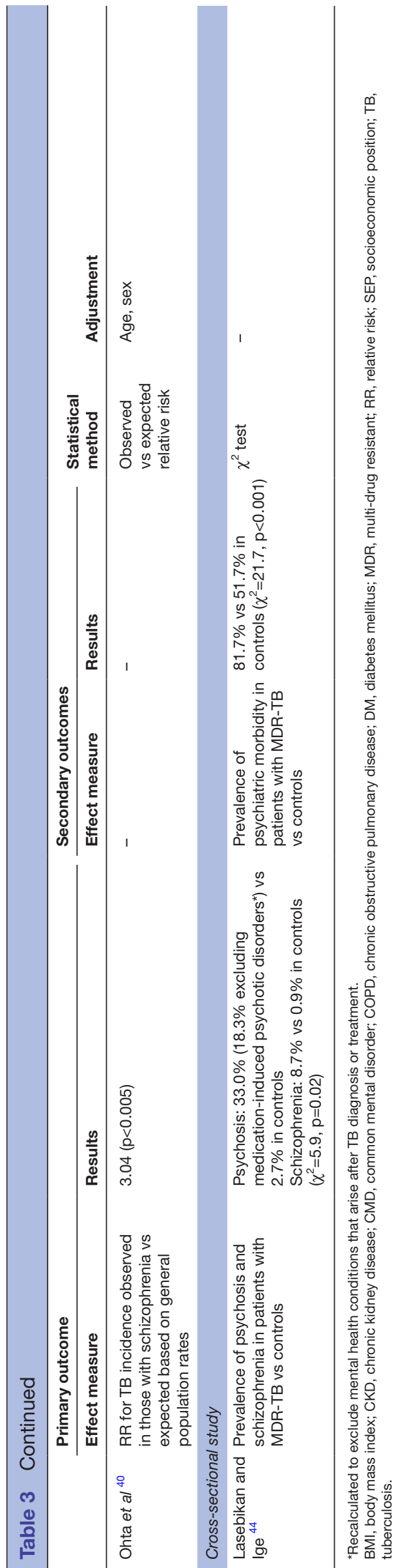

The schizophrenia studies are retrospective cohort studies, both finding that schizophrenia is significantly associated with TB risk. A large, high-quality study in Taiwan, using a nationwide database, reports an adjusted HR for TB incidence in those with schizophrenia versus controls of 1.52 (95\% CI 1.29 to $1.79, \mathrm{p}<0.001){ }^{39}$ The other, in Nagasaki, Japan uses registry data to identify TB incidence in those diagnosed with schizophrenia, and compares this to general population incidence. The reported relative risk (RR) for observed versus expected TB incidence is $3.04(\mathrm{p}<0.005) .{ }^{40}$ Both studies report a higher TB incidence rate in those with schizophrenia than in controls (figure 3).

A cross-sectional study investigates psychosis more generally among patients attending an MDR-TB clinic in Ibadan, Nigeria. This reports a prevalence of psychosis of $33.0 \%$ in patients with MDR-TB versus $2.7 \%$ in controls. When recalculated to exclude anti-TB medicationinduced psychotic disorders, this is a prevalence of $18.3 \%$ in patients with MDR-TB. This includes a prevalence of schizophrenia of $8.7 \%$ in TB patients versus $0.9 \%$ in controls $\left(\chi^{2}=5.9, \mathrm{p}=0.02\right){ }^{44}$

\section{DISCUSSION}

Included studies, synthesising data from 607184 individuals, show an association between mental health and TB. This includes robust evidence from cohort studies, all of which were based in Asia, demonstrating that depression and schizophrenia increase risk of TB disease. While the coexistence of poor mental health and TB is consistent with existing literature,${ }^{5}$ this is the first review to show that mental health is a risk factor for active TB.

Four population-based cohort studies are included, which are large studies using registry or insurance records to assess the relationship between medically coded depression or schizophrenia and TB disease in nationwide samples in Asia. Other studies, which are generally much smaller and cross-sectional in design, use questionnaire or interview mental health screening tools carried out in community or healthcare settings across Asia, South America and Africa. They therefore can detect less severe and undiagnosed conditions and can focus on specific, vulnerable populations such as the homeless. Whereas the cohort studies consider specific conditions such as depression or schizophrenia, most of the cross-sectional studies use screening tools that capture a wider range of mental health conditions.

Despite significant heterogeneity in study design and population, nine out of 10 included studies find a significant association between mental health and TB. In the one study that does not find a significant association, controls are individuals with prolonged respiratory symptoms, who might be expected to have a higher prevalence of depression than the general population. ${ }^{41}$ While not directly comparable, some of the observed variations may be attributed to different levels of adjustment for confounding; the cohort studies with more extensive 
Schizophrenia

Ohta et al 1988

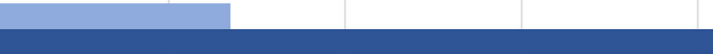

0

20

40

60

80

100

TB incidence rate (per 3251 persons)

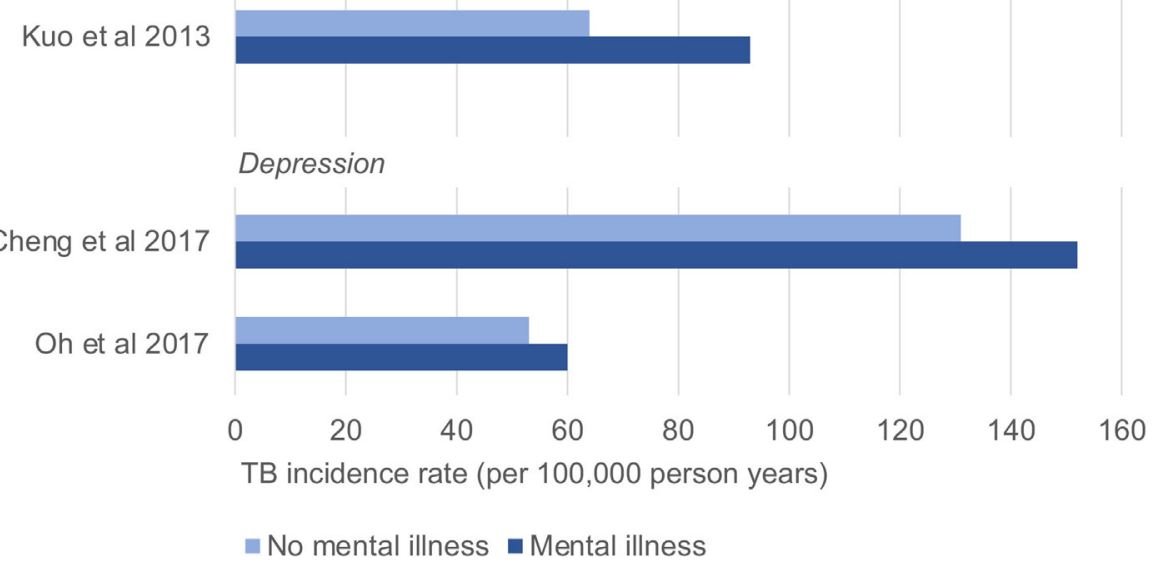

Figure 3 TB incidence rate reported in longitudinal studies. TB, tuberculosis.

adjustment for confounders ${ }^{37} 39$ show smaller effect sizes than their counterparts with less or no adjustment for confounders. ${ }^{3640}$

A key strength of this study is that, through undertaking an exhaustive search of the literature, it brings together a large sample of over 600000 individuals. By synthesising evidence from a range of study designs, we collate all the evidence on this topic and analyse the findings holistically. Nevertheless, this review has certain limitations. Future reviews could focus on LTBI, CNS TB and TB-HIV coinfection, which were excluded from this study. Moreover, the heterogeneity in study populations and methods made drawing direct comparisons between studies difficult and made meta-analysis inappropriate. For example, it was not possible to directly compare measures of effect generated from rates (HRs) with those generated from risks (RRs/ORs).

The nature of available evidence means that some caution is required when drawing conclusions. Studies addressing the association between mental health and TB are of variable design and quality, with four large studies (three cohort and one cross-sectional) accounting for 99\% of included participants. ${ }^{36} 373945$ Three included studies were classified as low quality; however, these only account for $0.001 \%$ of the total sample size (773 participants). ${ }^{38434}$ Only the cohort studies can establish temporality to provide compelling evidence that mental health precedes and acts as a risk factor for $\mathrm{TB}$, and these are the highest quality studies. ${ }^{36} 373940$ However, the cohort studies only cover depression and schizophrenia and are all from Asia. Further cohort studies are required to confirm whether these findings hold true for other mental illnesses in other global regions.

The study findings increase our understanding of TB risk factors, yet further research is needed to elucidate the pathways by which mental health may increase TB incidence, causally or via associations between mental health and other risk factors for TB such as alcohol/drug use, homelessness, incarceration, physical comorbidities and poverty. The relationships between mental health, $\mathrm{TB}$ and social risk factors are multi-directional, meaning that complex conceptual frameworks will be needed to understand the observed associations.

The existence of plausible immune mechanisms supports a causal explanation, with evidence that psychosocial stressors are associated with immune biomarkers relevant to $\mathrm{TB}^{48}$ Mental illnesses including depression are associated with various immunological changes which could increase susceptibility to $\mathrm{TB}^{14}{ }^{49}$; however, the key neuroendocrine and immunological pathways involved are unknown. In addition, mental health could be one factor influencing risk of progression from LTBI to active disease. Understanding the pathways that connect mental health and the immune response to TB may guide the development of host-directed approaches, for example, to prevent reactivation of LTBI.

The findings have implications for policy and clinical practice. The Lancet Commission on TB recommends that to achieve a TB-free world, populations at high risk must be reached and brought into care. ${ }^{50}$ The evidence presented here suggests that those suffering from mental illnesses, in particular depression and schizophrenia, could constitute such a high-risk group for active case-finding and treatment. This group could benefit from a holistic approach, integrating services for mental health and TB to facilitate rapid diagnosis and treatment of TB disease and LTBI, as well as providing better mental health support for individuals with TB. As such, the WHO End TB Strategy 2015-2035 recommends that treatment for TB and mental health is brought together. ${ }^{51}$ Providing high-quality mental health support and ensuring treatment adherence for TB both require substantial engagement with patients, so 
leveraging this contact to provide holistic care could prove effective. $^{52}$

In addition, the finding that mental illnesses constitute risk factors for TB suggests that tackling poor mental health and its underlying drivers may reduce TB incidence. In LMICs with a high TB incidence, poverty is consistently associated with common mental illnesses. ${ }^{53}$ Social protection schemes that lift individuals out of poverty are known to improve mental health and reduce TB risk factors ${ }^{54-56}$ suggesting that tackling poverty and associated poor mental health through investment in wider social policies could help reduce TB incidence. ${ }^{57}$

\section{Conclusion}

We find evidence that mental health is a risk factor for active TB. There is robust evidence from cohort studies based in Asia that depression and schizophrenia increase incidence of TB disease. This data, in combination with evidence from cross-sectional studies, identifies individuals with mental illnesses as a high-risk population for clinical TB that could be targeted for screening and treatment. This highlights the need for integrated programmes providing care for mental health and TB and suggests that interventions that tackle mental illnesses and their underlying drivers may help reduce TB incidence globally.

Contributors SEH and DB developed the concept and study design. SEH, AD and KR carried out screening, data extraction, critical appraisal and analysis. SEH is responsible for the overall content as guarantor. All authors (SEH, AD, KR, LN, ACS, $\mathrm{DB}, \mathrm{SH}, \mathrm{JSF})$ contributed to critical review of data and manuscript writing.

Funding SEH and AD are supported by Medical Research Council PhD studentships (MR/N013638/1), and KR receives funding from the Rosetrees Trust (M775). SH is supported by the National Institute for Health Research (NIHR Advanced Fellowship NIHR300072) and the Academy of Medical Sciences (SBF005\1111), and the European Society for Clinical Microbiology and Infectious Diseases (ESCMID) through an ESCMID Study Group for Infections in Travellers and Migrants (ESGITM)/ESCMID Study Group for Mycobacterial Infection (ESGMYC) research grant. LN receives funding from the Academy of Medical Sciences (SBF005\1047), the Medical Research Council/Economic and Social Research Council/Arts and Humanities Research Council (MR/T046732/1), and the Medical Research Council (MR/V027549/1). ACS is supported by the US National Institute of Mental Health (K01 MH104514).

Competing interests None declared.

Patient consent for publication Not applicable.

Ethics approval This study does not involve human participants.

Provenance and peer review Not commissioned; externally peer reviewed.

Data availability statement All data relevant to the study are included in the article or uploaded as supplementary information.

Supplemental material This content has been supplied by the author(s). It has not been vetted by BMJ Publishing Group Limited (BMJ) and may not have been peer-reviewed. Any opinions or recommendations discussed are solely those of the author(s) and are not endorsed by BMJ. BMJ disclaims all liability and responsibility arising from any reliance placed on the content. Where the content includes any translated material, BMJ does not warrant the accuracy and reliability of the translations (including but not limited to local regulations, clinical guidelines, terminology, drug names and drug dosages), and is not responsible for any error and/or omissions arising from translation and adaptation or otherwise.

Open access This is an open access article distributed in accordance with the Creative Commons Attribution 4.0 Unported (CC BY 4.0) license, which permits others to copy, redistribute, remix, transform and build upon this work for any purpose, provided the original work is properly cited, a link to the licence is given, and indication of whether changes were made. See: https://creativecommons.org/ licenses/by/4.0/.
ORCID iDs

Sally E Hayward http://orcid.org/0000-0002-4105-0990 Anna Deal http://orcid.org/0000-0001-6168-6542

Kieran Rustage http://orcid.org/0000-0003-1599-7635

Laura B Nellums http://orcid.org/0000-0002-2534-6951

Sally Hargreaves http://orcid.org/0000-0003-2974-4348

Jon S Friedland http://orcid.org/0000-0001-7789-9649

\section{REFERENCES}

1 World Health Organization. Global tuberculosis report 2020. Geneva: WHO, 2020.

2 Mathers CD, Loncar D. Projections of global mortality and burden of disease from 2002 to 2030. PLoS Med 2006;3:e442.

3 Prince M, Patel V, Saxena S, et al. No health without mental health. Lancet 2007;370:859-77.

4 Wang J, Wu X, Lai W, et al. Prevalence of depression and depressive symptoms among outpatients: a systematic review and metaanalysis. BMJ Open 2017;7:e017173.

5 Doherty AM, Kelly J, McDonald C, et al. A review of the interplay between tuberculosis and mental health. Gen Hosp Psychiatry 2013;35:398-406.

6 Sweetland A, Oquendo M, Wickramaratne P, et al. Depression: a silent driver of the global tuberculosis epidemic. World Psychiatry 2014;13:325-6.

7 Ruiz-Grosso P, Cachay R, de la Flor A, et al. Association between tuberculosis and depression on negative outcomes of tuberculosis treatment: a systematic review and meta-analysis. PLoS One 2020;15:e0227472

8 Pachi A, Bratis D, Moussas G, et al. Psychiatric morbidity and other factors affecting treatment adherence in pulmonary tuberculosis patients. Tuberc Res Treat 2013;2013:489865

9 Rock RB, Olin M, Baker CA, et al. Central nervous system tuberculosis: pathogenesis and clinical aspects. Clin Microbiol Rev 2008;21:243-61.

10 Public Health England. Tuberculosis in England: 2019 report. London: PHE, 2019.

11 Fazel S, Khosla V, Doll H, et al. The prevalence of mental disorders among the homeless in Western countries: systematic review and meta-regression analysis. PLoS Med 2008;5:e225.

12 Jané-Llopis E, Matytsina I. Mental health and alcohol, drugs and tobacco: a review of the comorbidity between mental disorders and the use of alcohol, tobacco and illicit drugs. Drug Alcohol Rev 2006;25:515-36.

13 Priebe S, Giacco D, El-Nagib R. Public health aspects of mental health among migrants and refugees: a review of the evidence on mental health care for refugees, asylum seekers and irregular migrants in the who European region (health evidence network (hen) synthesis report 47. Copenhagen: WHO Regional Office for Europe, 2016.

14 Sweetland AC, Kritski A, Oquendo MA, et al. Addressing the tuberculosis-depression syndemic to end the tuberculosis epidemic. Int J Tuberc Lung Dis 2017;21:852-61.

15 Lee GE, Scuffell J, Galea JT, et al. Impact of mental disorders on active TB treatment outcomes: a systematic review and metaanalysis. Int J Tuberc Lung Dis 2020;24:1279-84.

16 Munro SA, Lewin SA, Smith HJ, et al. Patient adherence to tuberculosis treatment: a systematic review of qualitative research. PLoS Med 2007;4:e238.

17 Alene KA, Clements ACA, McBryde ES, et al. Mental health disorders, social stressors, and health-related quality of life in patients with multidrug-resistant tuberculosis: a systematic review and meta-analysis. J Infect 2018;77:357-67.

18 Duko B, Bedaso A, Ayano G. The prevalence of depression among patients with tuberculosis: a systematic review and meta-analysis. Ann Gen Psychiatry 2020;19:30.

19 Duko B, Dana LM, Ayano G. Psychological distress among TB patients in sub-Saharan Africa. Int J Tuberc Lung Dis 2020:24:1200-4.

20 Glaser R, Kiecolt-Glaser JK. Stress-Induced immune dysfunction: implications for health. Nat Rev Immunol 2005;5:243-51.

21 Zhang K, Wang X, Tu J, et al. The interplay between depression and tuberculosis. J Leukoc Biol 2019;106:749-57.

22 Mason PH, Sweetland AC, Fox GJ, et al. Tuberculosis and mental health in the Asia-Pacific. Australasian Psychiatry 2016;24:553-5.

23 Janse Van Rensburg A, Dube A, Curran R, et al. Comorbidities between tuberculosis and common mental disorders: a scoping review of epidemiological patterns and person-centred care 
interventions from low-to-middle income and BRICS countries. Infect Dis Poverty 2020;9:4.

24 Chandra M, Rana P, Chandra K, et al. Tuberculosis - Depression syndemic: A public health challenge. Indian J Tuberc 2019;66:197-202.

25 de Araújo GS, Pereira SM, dos Santos DN. Revisão sobre tuberculose E transtornos mentais comuns. Revista Eletrônica Gestão \& Saúde 2014;5:716-26.

26 Moher D, Liberati A, Tetzlaff J, et al. Preferred reporting items for systematic reviews and meta-analyses: the PRISMA statement. PLoS Med 2009;6:e1000097.

27 Ouzzani M, Hammady H, Fedorowicz Z, et al. Rayyan-a web and mobile APP for systematic reviews. Syst Rev 2016;5:210.

28 World Health Organization. The ICD-10 classification of mental and behavioural disorders: diagnostic criteria for research. Geneva: WHO, 1993.

29 Lönnroth K, Williams BG, Stadlin S, et al. Alcohol use as a risk factor for tuberculosis - a systematic review. BMC Public Health 2008;8:289

30 Imtiaz S, Shield KD, Roerecke M, et al. Alcohol consumption as a risk factor for tuberculosis: meta-analyses and burden of disease. Eur Respir J 2017;50:1700216.

31 Deiss RG, Rodwell TC, Garfein RS. Tuberculosis and illicit drug use: review and update. Clin Infect Dis 2009;48:72-82.

32 World Health Organization. Definitions and reporting framework for tuberculosis - 2013 revision (updated December 2014 and January 2020. Geneva: WHO, 2020.

33 Cochrane Effective Practice and Organisation of Care (EPOC). Data collection form. EPOC resources for review authors, 2017. Available: http://epoc.cochrane.org/epoc-specific-resources-review-authors [Accessed 28 Mar 2018].

34 Critical Appraisal Skills Programme. Casp checklists, 2018. Available: https://casp-uk.net/casp-tools-checklists/ [Accessed 28 Mar 2018].

35 EpiNet. AXIS - appraisal tool. Available: http://www.epinet.net/AXISAppraisal-Tool [Accessed 25 Mar 2018].

$36 \mathrm{Oh} \mathrm{KH}$, Choi H, Kim EJ, et al. Depression and risk of tuberculosis: a nationwide population-based cohort study. Int J Tuberc Lung Dis 2017;21:804-9.

37 Cheng K-C, Liao K-F, Lin C-L, et al. Increased risk of pulmonary tuberculosis in patients with depression: a cohort study in Taiwan. Front Psychiatry 2017;8:235.

38 Srivastava D, Gupta LN, Vyas JN. Psychological aspects of pulmonary tuberculosis. Indian J Clin Psychol 1983;10:165-9.

39 Kuo S-C, Chen Y-T, Li S-Y, et al. Incidence and outcome of newlydiagnosed tuberculosis in schizophrenics: a 12-year, nationwide, retrospective longitudinal study. BMC Infect Dis 2013;13:351.

40 Ohta Y, Nakane Y, Mine M, et al. The epidemiological study of physical morbidity in schizophrenics -2 . association between schizophrenia and incidence of Tuberculosis-. Psychiatry Clin Neurosci 1988:42:41-7.

41 Castro-Silva KMde, Carvalho AC, Cavalcanti MT, et al. Prevalence of depression among patients with presumptive pulmonary tuberculosis in Rio de Janeiro, Brazil. Braz J Psychiatry 2019;41:316-23.
42 de Araújo GS, Pereira SM, dos Santos DN, et al. Common mental disorders associated with tuberculosis: a matched case-control study. PLoS One 2014;9:e99551.

43 Hernández Sarmiento JM, Correa N, Correa M, et al. Tuberculosis among homeless population from medellin, Colombia: associated mental disorders and socio-demographic characteristics. J Immigr Minor Health 2013;15:693-9.

44 Lasebikan VO, Ige OM. Prevalence of psychosis in tuberculosis patients and their nontuberculosis family contacts in a multidrug treatment-resistant treatment center in Nigeria. Gen Hosp Psychiatry 2015;37:542-7.

45 Koyanagi A, Vancampfort D, Carvalho AF, et al. Depression comorbid with tuberculosis and its impact on health status: cross-sectional analysis of community-based data from 48 low- and middle-income countries. BMC Med 2017:15:209.

46 Kroenke K, Spitzer RL, Williams JB. The PHQ-9: validity of a brief depression severity measure. J Gen Intern Med 2001;16:606-13.

47 World Health Organization. Composite international diagnostic interview (CIDI): core version 1.1. Geneva, Switzerland: World Health Organization, 1993.

48 Hayward SE, Dowd JB, Fletcher $\mathrm{H}$, et al. A systematic review of the impact of psychosocial factors on immunity: implications for enhancing BCG response against tuberculosis. SSM Popul Health 2020;10:100522.

49 Miller AH. Depression and immunity: a role for T cells? Brain Behav Immun 2010;24:1-8.

50 Reid MJA, Arinaminpathy N, Bloom A, et al. Building a tuberculosisfree world: the Lancet Commission on tuberculosis. The Lancet 2019;393:1331-84.

51 World Health Organization. Implementing the end TB strategy: the essentials. Geneva: WHO, 2015

52 Sweetland AC, Galea J, Shin SS, et al. Integrating tuberculosis and mental health services: global receptivity of national tuberculosis program directors. Int J Tuberc Lung Dis 2019;23:600-5.

53 Lund C, Breen A, Flisher AJ, et al. Poverty and common mental disorders in low and middle income countries: A systematic review. Soc Sci Med 2010;71:517-28.

54 Ozer EJ, Fernald LC, Weber A. Does alleviating poverty affect mothers' depressive symptoms? A quasi-experimental investigation of Mexico's Oportunidades programme. Int J Epidemiol 2011;40:1093/ije/dyr103:1565-76.

55 Powell-Jackson T, Pereira SK, Dutt V, et al. Cash transfers, maternal depression and emotional well-being: quasi-experimental evidence from India's Janani Suraksha Yojana programme. Soc Sci Med 2016;162:210-8.

56 Boccia D, Hargreaves J, Lönnroth K, et al. Cash transfer and microfinance interventions for tuberculosis control: review of the impact evidence and policy implications. Int J Tuberc Lung Dis 2011;15:37-49.

57 Shete PB, Reid M, Goosby E. Message to world leaders: we cannot end tuberculosis without addressing the social and economic burden of the disease. Lancet Glob Health 2018;6:e1272-3. 